\title{
Frequency Modulation Methods for Diffusion and Adsorption Measurements in Porous Solids
}

\author{
Sebastián C. Reyes,* John H. Sinfelt, Gregory J. DeMartin, and Richard H. Ernst \\ Corporate Research Laboratories, Exxon Research and Engineering Company, Route 22 East, \\ Annandale, New Jersey 08801 \\ Enrique Iglesia \\ Department of Chemical Engineering, University of California at Berkeley, Berkeley, California 94720 \\ Received: April 5, 1996; In Final Form: September 18, $1996^{\otimes}$
}

\begin{abstract}
Effective diffusivities and adsorption uptakes have been measured in mesoporous silica particles using frequency-modulated perturbation methods. Intraparticle diffusivities in the Knudsen regime were measured for $\mathrm{N}_{2}, \mathrm{Xe}, \mathrm{Xe} / \mathrm{N}_{2}$ mixtures, and isobutane at low pressure and several temperatures. The measured diffusivities show the correct dependence on temperature and molecular weight. The frequency response data show that, even above ambient temperatures, equilibrated adsorption occurs concurrently with diffusion processes. The high sensitivity of our experiments permits accurate measurements of the physisorption of $\mathrm{N}_{2}, \mathrm{Xe}$, and isobutane on silica at very low surface coverages, with heats of adsorption of 2.2, 3.4, and $5.6 \mathrm{kcal} / \mathrm{mol}$, respectively. The entropies of adsorption for $\mathrm{N}_{2}, \mathrm{Xe}$, and isobutane were determined to be $-16.4,-17.1$, and $-18.9 \mathrm{cal} /$ mol K, respectively, which are consistent with the loss of one degree of translational freedom. This study extends previous applications of frequency modulation methods to mesoporous solids useful as adsorbents and catalyst supports. These extensions were achieved by using a wider experimental frequency range and by developing new theoretical response functions for concurrent adsorption and diffusion processes.
\end{abstract}

\section{Introduction}

The pressure response of a closed system to a small fluctuation in its volume provides information about the rate processes occurring within porous solids contained within the system. When the volume fluctuations are small, the system equilibrium is not significantly perturbed and the theoretical interpretation of the response is simpler. Frequency modulation methods have been used previously to obtain kinetic parameters for chemical reactions and adsorption-desorption steps on surfaces and to measure effective diffusivities within microporous solids. ${ }^{1-9}$

Coupled adsorption and diffusion processes with different characteristic time scales can be studied at typical conditions of catalytic reactions by varying the frequency of the applied perturbations. In general, these rate processes cannot be adequately resolved by pulse or step transient methods, by temperature-programmed desorption, or by elution chromatography. Previous applications of frequency response methods in heterogeneous catalysis have been limited by experimental restrictions in accessible frequency range and by the lack of theoretical descriptions that properly account for the coupling of several concurrent dynamic processes. ${ }^{9}$

In this work, we extend experimental and theoretical frequency response methods to study diffusion and adsorption within mesoporous silica particles. Specifically, we present new analytical expressions for the system transfer functions, which are required in order to extract effective diffusivities and adsorption-desorption constants from the measured system response. These transfer functions characterize the pressure response of the closed system to a small fluctuation in its volume. The rates of the underlying adsorption and transport processes are sensitive to the properties of the solid surfaces and to the morphology of the intraparticle pore voids. In frequency response experiments, the coupling between adsorp-

* Corresponding author (fax 908-730-3301; internet screyes@erenj.com).

${ }^{\otimes}$ Abstract published in Advance ACS Abstracts, January 1, 1997. tion and transport is particularly important for high surface area materials, because the number of molecules exchanging between the surface and the gas phase within the pores in the adsorption process, even at low surface coverages, can greatly exceed those exchanging between the pore volume and the external gas phase in the diffusion process. We show that the proper accounting of equilibrated adsorption is essential in order to obtain accurate diffusion coefficients within porous solids.

\section{Background}

Frequency modulation methods exploit the change in amplitude and phase produced by a dynamic system that is perturbed periodically around its equilibrium point. The resulting amplitude change (attenuation) and phase shift (lag) are directly related to the dynamics and capacities of the processes governing the return of the system to its equilibrium point. For example, a small sinusoidal variation in the volume of a closed system containing a porous nonadsorbing solid results in a sinusoidal pressure response. The extent of attenuation and lag of the response is determined by the effective diffusivity (dynamics) and by the pore volume within the porous solid (capacity). When adsorption-desorption occurs concurrently with diffusion, the resulting pressure response depends also on the kinetics of adsorption (dynamics) and on the changes in surface coverage caused by the applied perturbation (capacity). ${ }^{9}$

A graphical description of the frequency modulation approach is shown in Figure 1. At each frequency $\omega$, the system volume is perturbed sinusoidally with amplitude $v$ (solid line). The system pressure responds in a sinusoidal manner with a fluctuation amplitude $p$ (dotted line), which is lower than that corresponding to $v$ at equilibrium and is delayed by an angle $\varphi$. The capacities and dynamics of the underlying processes are obtained by fitting the experimental transfer function obtained from the measured quantities $v, p$, and $\varphi$ to the theoretical transfer function $H(\mathrm{i} \omega)$. The transfer function $H(\mathrm{i} \omega)$ is an analytical expression in frequency domain, which contains the parameters to be determined. Its magnitude measures the 


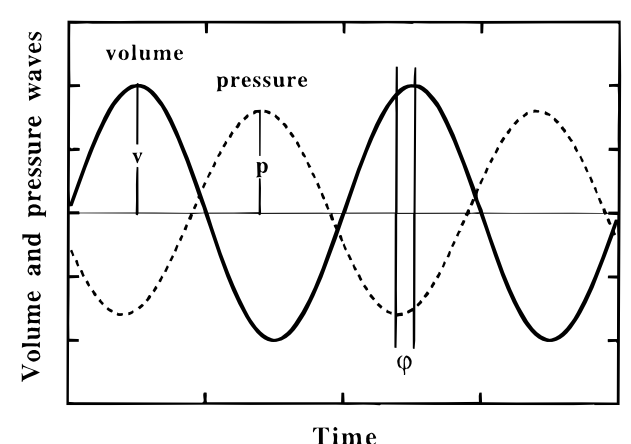

Figure 1. Input volume perturbation and output pressure response in frequency response experiments.

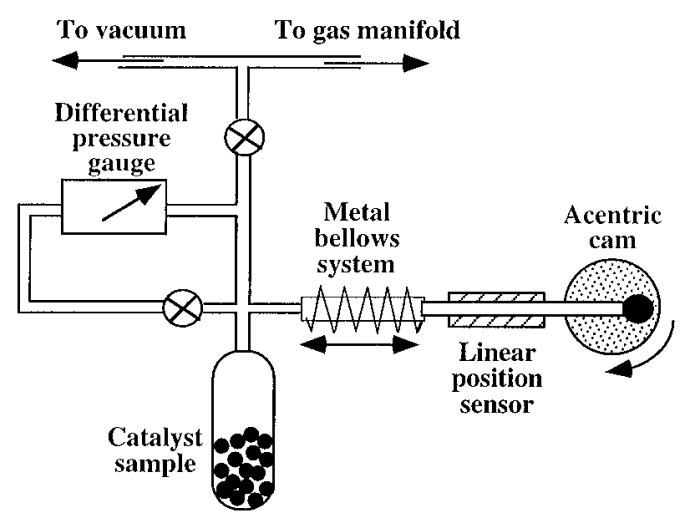

Figure 2. Schematic diagram of frequency response apparatus.

ratio of the pressure response to the volume perturbation $(|H|$ $=p / v)$ and also quantifies the phase lag $\left(\varphi=\tan ^{-1}[\operatorname{Im}(H) / \operatorname{Re}-\right.$ $(H)])$. Transfer functions are uniquely determined by the constitutive equations describing the chemical and transport processes responsible for the observed dynamic response. The derivation of these transfer functions is best approached via perturbation methods that exploit $v$ as the small parameter in the frequency response experiments. A detailed description of the mathematical perturbation methods used to derive the transfer functions has been presented elsewhere. ${ }^{9}$

\section{Methods}

3.1. Experimental Methods. The experimental apparatus (Figure 2) allows diffusivity and adsorption measurements at input perturbation frequencies between 0.05 and $10 \mathrm{~Hz}$. The system volume $\left(V=141.91 \mathrm{~cm}^{3}\right)$ can be sinusoidally modulated by $1.38 \%\left(\Delta V=1.96 \mathrm{~cm}^{3}\right)$ using a metal bellows. The apparatus is constructed of quartz and Pyrex, and the system volume is contained within a controlled temperature box (223$400 \mathrm{~K})$. Higher temperatures can be achieved during pretreatment by direct resistive heating of the sample cell. The geometry of the system volume minimizes hydrodynamic delays that are not related to the dynamics of intraparticle diffusion and adsorption. This volume contains a sample cell, a steel bellows driven by a rotary motor with an acentric cam, and a differential pressure gauge (MKS Model $223 \mathrm{BD}, 0-130 \mathrm{~Pa}$ ). Dynamic system pressures of $10^{-9} \mathrm{~Pa}$ can be routinely achieved by turbomolecular and mechanical vacuum pumps. The position of the bellows is determined by a linear variable differential transformer (Schaevitz Engineering), which measures the input volume perturbation. Data are acquired using LabTech Notebook software and a personal computer (Dell PC-AT 320). Turbo Pascal codes are used to control input voltages to the motor and to determine phase lags, amplitude attenuations, and characteristic response functions from pressure-volume experimental data.
Mesoporous silica spheres commonly used as catalyst supports were used in all experiments (Shell S980B, BET surface area $204 \mathrm{~m}^{2} / \mathrm{g}$; pore volume per unit mass of particles $=1.16$ $\mathrm{cm}^{3} / \mathrm{g}$; particle density $\rho_{\mathrm{p}}=0.58 \mathrm{~g} / \mathrm{cm}^{3} ; \mathrm{SiO}_{2}$ skeletal density $\rho_{\mathrm{s}}=2.3 \mathrm{~g} / \mathrm{cm}^{3}$; mean pore radius $r_{\mathrm{p}}=3.5 \mathrm{~nm}$, from $\mathrm{N}_{2}$ physisorption; mean particle radius $R_{0}=0.135 \mathrm{~cm}$, from optical microscopy). All experiments reported here were carried out using $16.5 \mathrm{~g}$ of silica particles at an equilibrium pressure of $2.13 \mathrm{kPa}$ (16 Torr). Increasing or decreasing the amount of silica particles did not change the frequency response measurements, and therefore it was concluded that bed-depth effects, such as those reported by Van-den-Begin and Rees, ${ }^{18}$ were not present in any of the experiments reported here. The samples were calcined at $873 \mathrm{~K}$ for $16 \mathrm{~h}$ and then loaded into the frequency response reactor cell. The samples were then heated to $573 \mathrm{~K}$ in dynamic vacuum for $2 \mathrm{~h}$ before frequency response measurements. $\mathrm{N}_{2}$ (Matheson, 99.998\%), isobutane (Matheson, 99.5\%), and Xe (Matheson, 99.995\%) gases were used without further purification.

3.2. Theoretical Methods. This section describes key steps in the development of theoretical transfer functions that quantify the amplitude attenuation $(p / v)$ and phase lag $(\varphi)$ observed in diffusion and adsorption experiments. In section 3.2.1, we first analyze adsorption-desorption processes on uniform surfaces (Langmuir adsorption). This analysis shows that frequency response experiments measure the relaxation time for adsorption and are sensitive to the local slope of the adsorption isotherm at the equilibrium pressure.

In section 3.2.2, we analyze Fickian diffusion of gases in nonadsorbing porous solids and show that frequency response methods measure pore volumes and effective diffusivities. This analysis of diffusion dynamics in frequency response experiments yields information exactly analogous to the effectiveness factors in catalytic reactions, where frequency in the former plays the role of the first-order kinetic constant in the latter. Finally, in section 3.2.3, we describe the frequency response characteristics of concurrent adsorption and diffusion processes. We first describe the general case, in which the rates of diffusion and adsorption are comparable, and then the case of rapid adsorption. Rapid (or equilibrated) adsorption is a case of particular interest for porous metal oxides, because quasiequilibrated physisorption processes can mask the true values of the effective diffusion coefficients even at very low surface coverages.

3.2.1. Adsorption. Let's consider a mass $m$ of porous adsorbent or catalyst particles contained within a closed system of equilibrium volume $V_{\mathrm{e}}$. The equilibrium volume is defined as the volume of the system minus the volume of particles, the latter excluding the pore volume $\left(V_{\mathrm{e}}=V-m / \rho_{\mathrm{s}}\right)$. For Langmuir adsorption, the concentration of adsorbed species changes with pressure according to

$$
\frac{\mathrm{d} n_{\mathrm{s}}}{\mathrm{d} t}=k_{\mathrm{a}} P\left(N-n_{\mathrm{s}}\right)-k_{\mathrm{d}} n_{\mathrm{s}}
$$

If the gas-surface exchange reaction is the only dynamic process with a relaxation frequency within the range covered by the frequency response experiments, then its transfer function $H(\mathrm{i} \omega)$ is given by ${ }^{9}$

$$
\begin{aligned}
& H(\mathrm{i} \omega)=\frac{1}{1+K_{\mathrm{s}} \lambda} \quad K_{\mathrm{s}}=\frac{m R T\left(\mathrm{~d} n_{\mathrm{s}} / \mathrm{d} P\right)_{P=P_{\mathrm{e}}}}{V_{\mathrm{e}}} \\
& \lambda(\mathrm{i} \Omega)=\frac{1}{1+\mathrm{i} \Omega}
\end{aligned}
$$




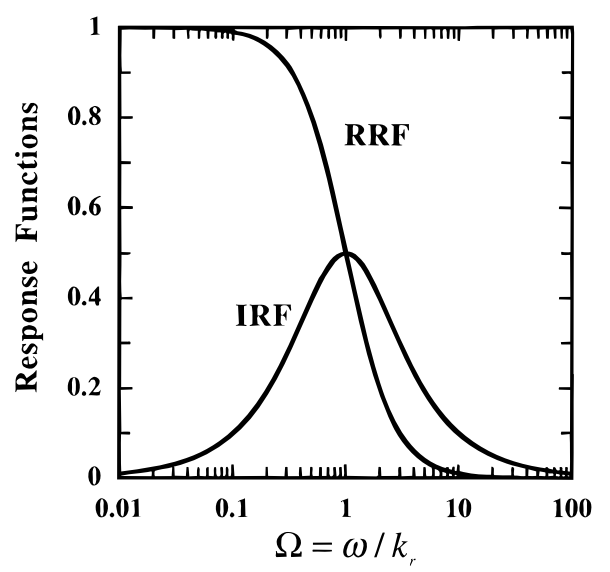

Figure 3. RRF and IRF components for adsorption processes (eq 4) $\left[K_{\mathrm{s}}=1\right]$.

In these equations, $\Omega=\omega / k_{\mathrm{r}}$ is the dimensionless frequency and $k_{\mathrm{r}}=k_{\mathrm{a}} P_{\mathrm{e}}+k_{\mathrm{d}}$ is the adsorption relaxation frequency. $K_{\mathrm{s}}$ and $\lambda$ are dimensionless quantities describing the capacity and the dynamics of the adsorption process, respectively. $K_{\mathrm{s}}$ measures the ratio of the volume of gas exchanged with the surface during one fluctuation to the equilibrium volume of the system, and $\lambda$ is a measure of the ratio of the characteristic modulation time to the adsorption relaxation time. Equation 2 clearly shows that $K_{\mathrm{s}}$ is proportional to the slope of the adsorption isotherm, evaluated at the equilibrium pressure $P_{\mathrm{e}}$. The transfer function $H(\mathrm{i} \omega)$ describes the amplitude attenuation $(|H|=p / v)$ and phase lag $\left(\varphi=\tan ^{-1}[\operatorname{Im}(H) / \operatorname{Re}(H)]\right)$ at each modulating frequency $\omega$. A least-squares fit of the data $(p / v$ and $\varphi$ ) allows the estimation of $K_{\mathrm{s}}$ and $k_{\mathrm{r}}$, the unknown parameters in eq 2 .

Previous theoretical treatments of adsorption ${ }^{2,3}$ described the frequency response behavior in terms of the so-called real (RRF) and imaginary (IRF) response functions. These RRF and IRF functions are uniquely related to the transfer function $H(\mathrm{i} \omega)$ by

$$
\mathrm{RRF}=\operatorname{Re}\left[\frac{1}{H(\mathrm{i} \omega)}-1\right] \quad \operatorname{IRF}=-\operatorname{Im}\left[\frac{1}{H(\mathrm{i} \omega)}-1\right]
$$

Substitution of $H(\mathrm{i} \omega)$ from eq 2 into eq 3 gives the RRF and IRF response functions for pure adsorption-desorption processes on uniform surfaces (Langmuir-type):

$$
\mathrm{RRF}_{\text {theory }}=\frac{K_{\mathrm{s}}}{1+\Omega^{2}} \quad \mathrm{IRF}_{\text {theory }}=\frac{K_{\mathrm{s}} \Omega}{1+\Omega^{2}}
$$

These equations are identical to those reported by Naphtali and Polinski $^{2}$ and Yasuda $^{3}$ for Langmuir adsorption. In cases where coupled dynamic processes occur, it is simpler to cast the system response in terms of a transfer function rather than RRF and IRF functions. In general, however, $H(\mathrm{i} \omega)$ and the RRF and IRF functions are uniquely related as shown in eq 3 .

To compare the theory to the experimental data, we need expressions for the RRF and IRF functions in terms of the measurable quantities $v, p$, and $\varphi$. Following the definition of $H=|H| \mathrm{e}^{\mathrm{i} \varphi}=(p / v) \mathrm{e}^{\mathrm{i} \varphi}$ and eq 3 , the experimental RRF and IRF functions are evaluated as

$$
\begin{array}{r}
\mathrm{RRF}_{\text {experiment }}=(v / p) \cos (\varphi)-1 \text { and } \\
\qquad \operatorname{IRF}_{\text {experiment }}=(v / p) \sin (\varphi)
\end{array}
$$

Equation 5 clearly shows that the only measurements needed in frequency response experiments are the values of $v, p$, and $\varphi$ at each frequency. Thus, the capacity parameter $K_{\mathrm{s}}$ and dynamic parameter $k_{\mathrm{r}}$ are obtained by comparing the theoretical (eq 4) and experimental (eq 5) RRF and IRF functions.

RRF and IRF functions are plotted against dimensionless frequency in Figure 3 in order to highlight the capacity and the dynamics of the adsorption process, respectively. For example, at low modulation frequencies $(\Omega \rightarrow 0)$, the RRF component approaches $K_{\mathrm{S}}$ asymptotically and the IRF component approaches zero. Thus, when the period of the perturbation is much longer than the adsorption relaxation time, the RRF provides a direct measure of the adsorption capacity of the solid as a function of pressure. The IRF component goes through a maximum when the period of the perturbation equals the adsorption relaxation time $(\Omega=1)$, a measure of the adsorption dynamics. At high frequencies $(\Omega \rightarrow \infty)$, both the RRF and the IRF approach zero.

3.2.2. Diffusion. In the absence of adsorption, the diffusional transport of a gas within the pores of spherical particles is governed by Fick's diffusion equation:

$$
\mathrm{d} C / \mathrm{d} t=D \nabla^{2} C
$$

If the particles are contained within a closed volume that is sinusoidally modulated around its equilibrium volume $V_{\mathrm{e}}$, the transfer function is given by

$$
H(\mathrm{i} \omega)=\frac{1}{1+K_{\mathrm{g}} \eta(\Phi)}
$$

where

$$
\begin{array}{r}
K_{\mathrm{g}}=\frac{m R T\left(\mathrm{~d} n_{\mathrm{g}} / \mathrm{d} P\right)_{P=P_{\mathrm{e}}}}{V_{\mathrm{e}}} \quad \eta(\Phi)=\frac{3}{\Phi}\left(\operatorname{coth}(\Phi)-\frac{1}{\Phi}\right) \\
\Phi^{2}=\frac{\mathrm{i} \omega R_{0}{ }^{2}}{D}
\end{array}
$$

If the gas molecules within the particles behave as an ideal gas, then the capacity parameter $K_{\mathrm{s}}$ can be rewritten as

$$
K_{\mathrm{g}}=\frac{V_{\mathrm{p}}}{V_{\mathrm{e}}}=\frac{m\left(\epsilon / \rho_{\mathrm{p}}\right)}{V_{\mathrm{e}}}
$$

In analogy to the adsorption case described in section 3.2.1, the transfer function for diffusion (eq 7) is also written in terms of a capacity parameter $K_{\mathrm{g}}$ and a dynamic parameter $\eta$. At low frequencies (where $\Phi \rightarrow 0$ and $\eta \rightarrow 1$ ), the transfer function provides a direct measure of the capacity parameter $K_{\mathrm{g}} . K_{\mathrm{g}}$ is proportional to the pore volume of the particles, which is the only "storage" capacity within a nonadsorbing porous solid. The quantity $D / R_{0}^{2}$ appearing in $\Phi^{2}$ is the diffusional relaxation frequency, which depends on the effective diffusivity and the diffusion distance in the porous particles.

The dynamic term $\eta$ has the same functional dependence on $\Phi$ as the effectiveness factor derived for first-order chemical reactions in spherical particles. ${ }^{12}$ In heterogeneous catalysis, $\Phi$ is called the Thiele modulus and it measures the ratio of the characteristic diffusion time to the characteristic reaction time for the chemical reaction. In frequency response experiments, $\Phi$ is the ratio of the characteristic diffusion time to the characteristic modulation time. Thus, $\omega$ in frequency response experiments plays a role analogous to the first-order kinetic constant in the Thiele treatment of heterogeneous catalysis. In physical terms, $\eta$ (eq 8) measures the fraction of the intraparticle pore volume that is invaded during the period of one perturba- 


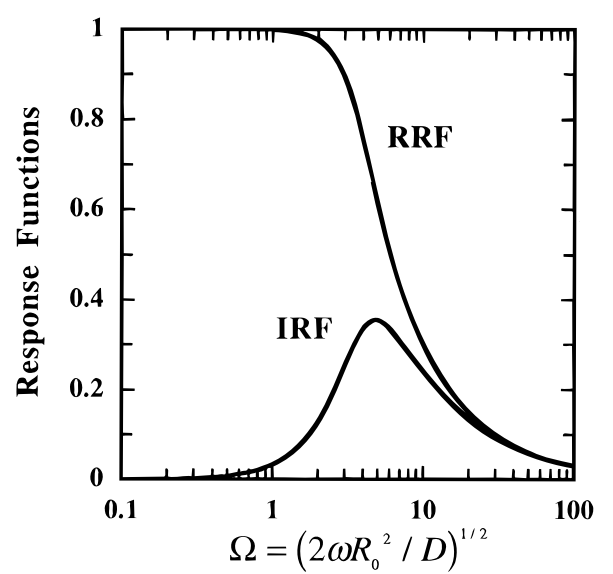

Figure 4. RRF and IRF components for diffusion processes (eqs 10 and 11) $\left[K_{\mathrm{g}}=1\right]$.

tion. This analogy with catalytic reactions is a very important result, because it implies that $\eta$ in other particle geometries (e.g., slabs and cylinders) is the same as the corresponding effectiveness factor $\eta(\Phi)$ derived for first-order catalytic reactions. Moreover, all renormalizations of the Thiele modulus $\Phi$ that seek a general representation of the effectiveness factor for arbitrary geometries can be extended to the dynamic quantity $\eta$ in frequency response experiments. ${ }^{12}$

Following eq 3, the transfer function for diffusion (eq 8) can also be written in terms of the RRF and IRF components as follows:

$$
\begin{gathered}
\mathrm{RRF}_{\text {theory }}=K_{\mathrm{g}} \frac{3}{\Omega}\left[\frac{\sinh (\Omega)-\sin (\Omega)}{\cosh (\Omega)-\cos (\Omega)}\right] \\
\mathrm{IRF}_{\text {theory }}=K_{\mathrm{g}} \frac{6}{\Omega}\left[\frac{1}{2}\left(\frac{\sinh (\Omega)+\sin (\Omega)}{\cosh (\Omega)-\cos (\Omega)}\right)-\frac{1}{\Omega}\right]
\end{gathered}
$$

Here, $\Omega=\left(2 \omega R_{0}^{2} / D\right)^{1 / 2}$ is the dimensionless frequency, and the diffusion coefficient $D$ and the capacity parameter $K_{\mathrm{g}}$ are the only unknown parameters estimated by minimizing the deviation between theoretical (eqs 10 and 11) and experimental response functions (eq 5). When plotted as a function of $\Omega$, the theoretical RRF and IRF functions display features similar to those for adsorption (Figure 4). The RRF and IRF curves for diffusion, however, do not cross and instead approach zero asymptotically and concurrently. The IRF goes through a maximum at a dimensionless frequency of $\Omega \approx 4.818$, a value obtained numerically from eq 11 . The RRF and IRF functions (eqs 10 and 11) are identical to those previously derived using a different approach. ${ }^{8}$

3.2.3. Coupled Adsorption and Diffusion. In the previous sections, one dynamic process (adsorption or diffusion) caused the delay and attenuation of the imposed perturbation. This section addresses the case of diffusion and adsorption processes occurring at comparable rates and invading two distinct reservoirs: the pore voids and the solid surface, respectively. This assumption applies well to mesoporous solids, with pores that are large compared to molecular sizes but are small enough to provide a large specific surface area; it is not valid for microporous solids, such as zeolites, where the dimensions of channels and cavities are comparable to those of diffusing molecules. In the latter case, the distinction between gas and adsorbed species cannot be made because molecules are always influenced by the properties of the confining surfaces.

When diffusion rates are comparable to adsorption rates, the material balances for the gas and adsorbed phases are described by

$$
\begin{gathered}
\frac{\partial C}{\partial t}+\rho_{\mathrm{p}} \frac{\partial n_{\mathrm{s}}}{\partial t}=D \nabla^{2} C \\
\frac{\partial n_{\mathrm{s}}}{\partial t}=k_{\mathrm{a}} P\left(N-n_{\mathrm{s}}\right)-k_{\mathrm{d}} n_{\mathrm{s}}
\end{gathered}
$$

A frequency response analysis of this coupled system of differential equations leads to the following transfer function: ${ }^{9}$

$$
H(\mathrm{i} \omega)=\frac{1}{1+\left(K_{\mathrm{g}}+K_{\mathrm{s}} \lambda\right) \eta(\Phi)}
$$

where

$$
\Phi^{2}=\frac{i \omega R_{0}^{2}}{D}\left[1+\alpha K_{\mathrm{s}} \lambda\right]
$$

The form of this transfer function (eq 14) is similar to those previously obtained for pure adsorption and pure diffusion, but includes now the coupling between them. In contrast with the pure diffusion case, the modulus $\Phi^{2}$ now contains an additional dimensionless parameter $\left(\alpha K_{\mathrm{s}} \lambda\right)$ that reflects the extra capacity imposed by adsorption on the diffusion process. This additional term is equal to the product of the capacity parameter $\left(K_{\mathrm{S}}\right)$ and the dynamic parameter $(\lambda)$ of the adsorption process, corrected by the geometric factor $\alpha=V_{\mathrm{e}} /\left(m / \rho_{\mathrm{p}}\right)$. The factor $\alpha$ simply renormalizes $K_{\mathrm{s}}$ to the volume of the particles instead of the volume of the system because the term $\Phi^{2}$ pertains to diffusion within each individual particle. As before, for spherical particles, the dynamic term $\eta(\Phi)$ for diffusion is given by eq 8.

According to eqs 2,8 , and 15 , when the period of the modulation frequency is much longer than the diffusion and adsorption relaxation times, both $\lambda$ and $\eta$ approach unity and the total capacity of the system equals the sum of the pore volume and the equilibrium adsorption uptake (see eq 14). When the period of the modulation is short, $\eta$ approaches zero because molecules cannot penetrate the particles during one perturbation period. As done previously for independent adsorption and diffusion processes, the transfer function for coupled diffusionadsorption can be expressed as RRF and IRF components. Unfortunately, the resulting analytical expressions are quite unwieldy and do not provide more insight than the transfer function itself. Numerical values of the RRF and IRF components can always be obtained straightforwardly by using eq 3 .

Two important limits of eq 14 occur when the rate of diffusional transport is rapid or slow compared to the rate of adsorption. The former situation can be described by the results of the previous sections because, effectively, the diffusion and adsorption processes become decoupled, leading to dynamics controlled by pure diffusion at high frequencies and pure adsorption at low frequencies. When the rate of adsorption is fast when compared to diffusional transport, the transfer function acquires diffusion-like characteristics. Here, $\lambda$ approaches unity and the transfer function becomes

$$
H(\mathrm{i} \omega)=\frac{1}{1+K^{\mathrm{app}} \eta(\Phi)}
$$

where

$$
\Phi^{2}=\frac{\mathrm{i} \omega R_{0}^{2}}{D^{\mathrm{app}}} \quad K^{\mathrm{app}}=K_{\mathrm{g}}+K_{\mathrm{s}} \quad D^{\mathrm{app}}=\frac{D}{1+\alpha K_{\mathrm{s}}}
$$

This transfer function (eq 16) has the same form as that for pure diffusion (eq 7), except that its capacity ( $\left.K^{\text {app}}\right)$ and effective 

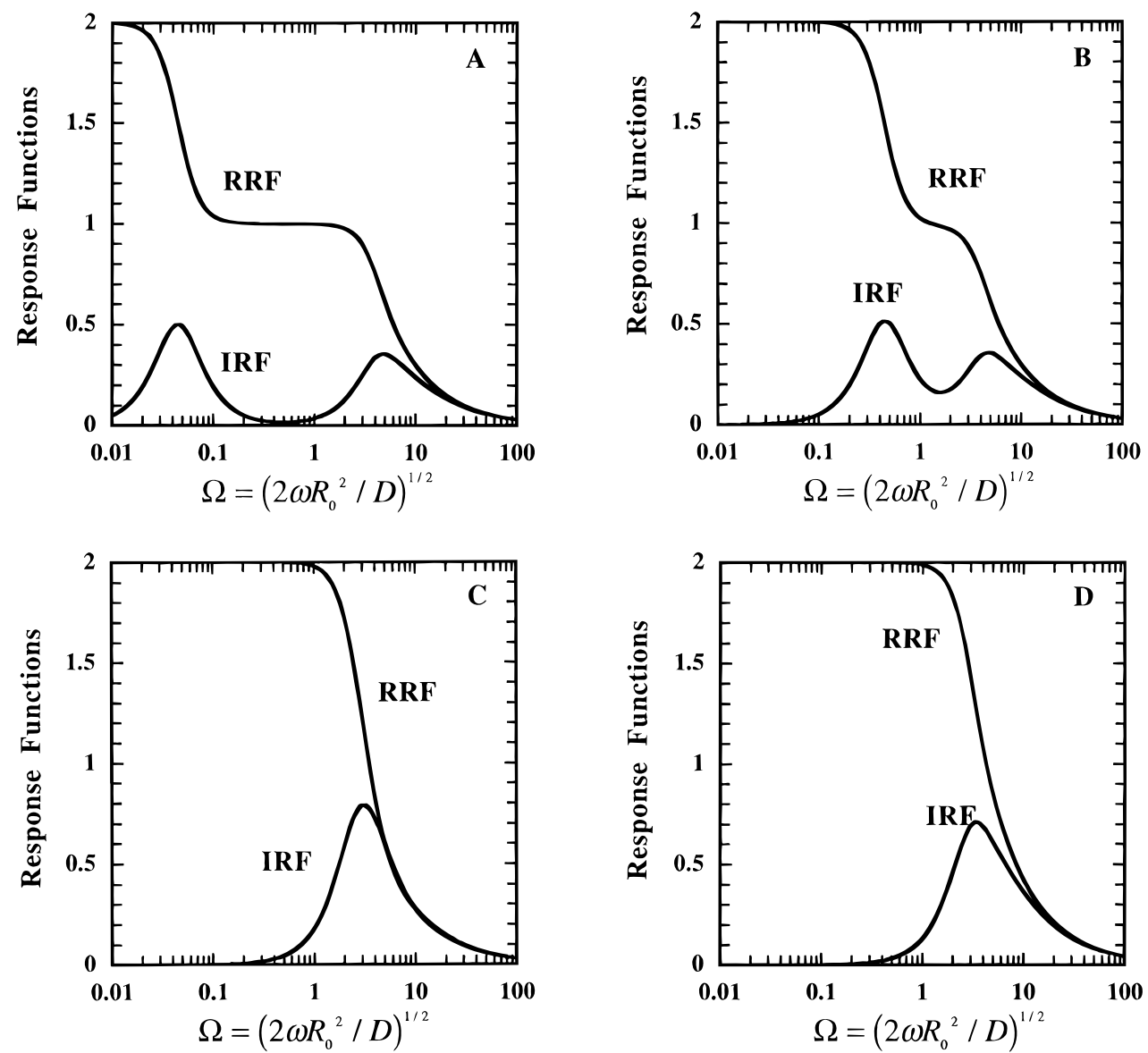

Figure 5. RRF and IRF components for coupled diffusion and adsorption processes (eqs 3, 14, and 15). Effect of increasing the adsorption relaxation frequency $k_{\mathrm{r}}(\mathrm{A}) 1 \times 10^{-5} \mathrm{~s}^{-1}$; (B) $=1 \times 10^{-3} \mathrm{~s}^{-1}$; (C) $1 \times 10^{-1} \mathrm{~s}^{-1}$; (D) $1 \times 10^{4} \mathrm{~s}^{-1}\left[K_{\mathrm{s}}=K_{\mathrm{g}}=1, \alpha=1, D / R_{0}^{2}=100 \mathrm{~s}^{-1}\right]$.

diffusivity ( $\left.D^{\text {app }}\right)$ become the apparent values given in eq 17. This diffusion-like behavior reflects how a fast adsorption process increases the storage capacity of the solid above that of the pore volume and how the maximum in the IRF function is displaced to lower frequencies. Figure 5 illustrates some key features of the response of coupled adsorption and diffusion processes. The RRF and IRF components were calculated for adsorption and diffusion processes of equal capacity but differing dynamics. Clearly, when adsorption is much slower than diffusion, the processes are essentially decoupled (Figure 5A); the supply of molecules by diffusion occurs much faster than the adsorption exchange. As adsorption rates increase and approach diffusion rates, the IRF adsorption peak begins to merge with the diffusion peak (Figure 5B,C). Ultimately, when adsorption is very fast, the response functions become identical to those for pure diffusion (Figure 5D). Figure 5D clearly shows that a diffusion-like frequency response may hide a fast adsorption process (compare to Figure 4). A fast adsorption process is present if the magnitude of the low-frequency limit of the RRF function exceeds the expected pore volume capacity $V_{\mathrm{p}} / V_{\mathrm{e}}$ of the solid. Also, according to eq 17, a fast adsorption process displaces the maximum of the IRF function to lower frequencies by the factor $\left(1+\alpha K_{\mathrm{s}}\right)^{1 / 2}$ (e.g., $\Omega$ decreases from a value of 4.82 in Figure 4 to 3.41 in Figure 5D) for the same value of $D$. These tests will be used in the next section to determine the extent of rapid adsorption that occurs concurrently with the diffusion processes.

This section has specifically addressed the derivation of response functions for coupled adsorption and diffusion processes that apply to the conditions of our experiments. A related development that applies to a more comprehensive case of coupled adsorption and diffusion in bidisperse porous structures subject to external mass transfer limitations has been recently presented by Jordi and Do. ${ }^{18}$ Their work illustrates the importance of developing fundamental models that can capture all dynamic processes that influence the measured response.

\section{Results and Discussion}

4.1. Diffusion of $\mathbf{N}_{2}$ in Mesoporous Silica. The frequency response of a system containing $\mathrm{N}_{2}$ and silica particles to sinusoidal volume perturbations of varying frequency $(0.1-6$ $\mathrm{Hz}$ ) is shown in Figure 6. The measurements were carried out at $308,348,398$, and $573 \mathrm{~K}$ in order to study the effect of temperature on the diffusion coefficients and to minimize nitrogen physisorption. As suggested by eq 5, the data $v, p$, and $\varphi$ were plotted as RRF and IRF components. The response functions show diffusion-like characteristics similar to those illustrated in Figures 4 and 5D. At low frequencies, pressure fluctuations are sufficiently slow that nitrogen concentration gradients do not develop within the pore structure of the particles. At high frequencies, the RRF and IRF approach each other and decrease toward zero, signaling that intraparticle nitrogen concentration gradients develop as the external fluctuations become more rapid.

Figure 6 shows that the maximum in the IRF shifts to higher frequencies and the height of the RRF decreases in magnitude as temperature increases. The frequency shift reflects a diffusivity increase with temperature; the amplitude attenuation reflects a decrease in physisorption uptakes as temperature increases. At very low frequencies, the height of the RRF (or total nitrogen storing capacity) decreases from 0.2 at $308 \mathrm{~K}$ (Figure 5A) to about 0.15 at $573 \mathrm{~K}$ (Figure 5B). Only at 573 $\mathrm{K}$ is this capacity consistent with the pore volume of the particles 

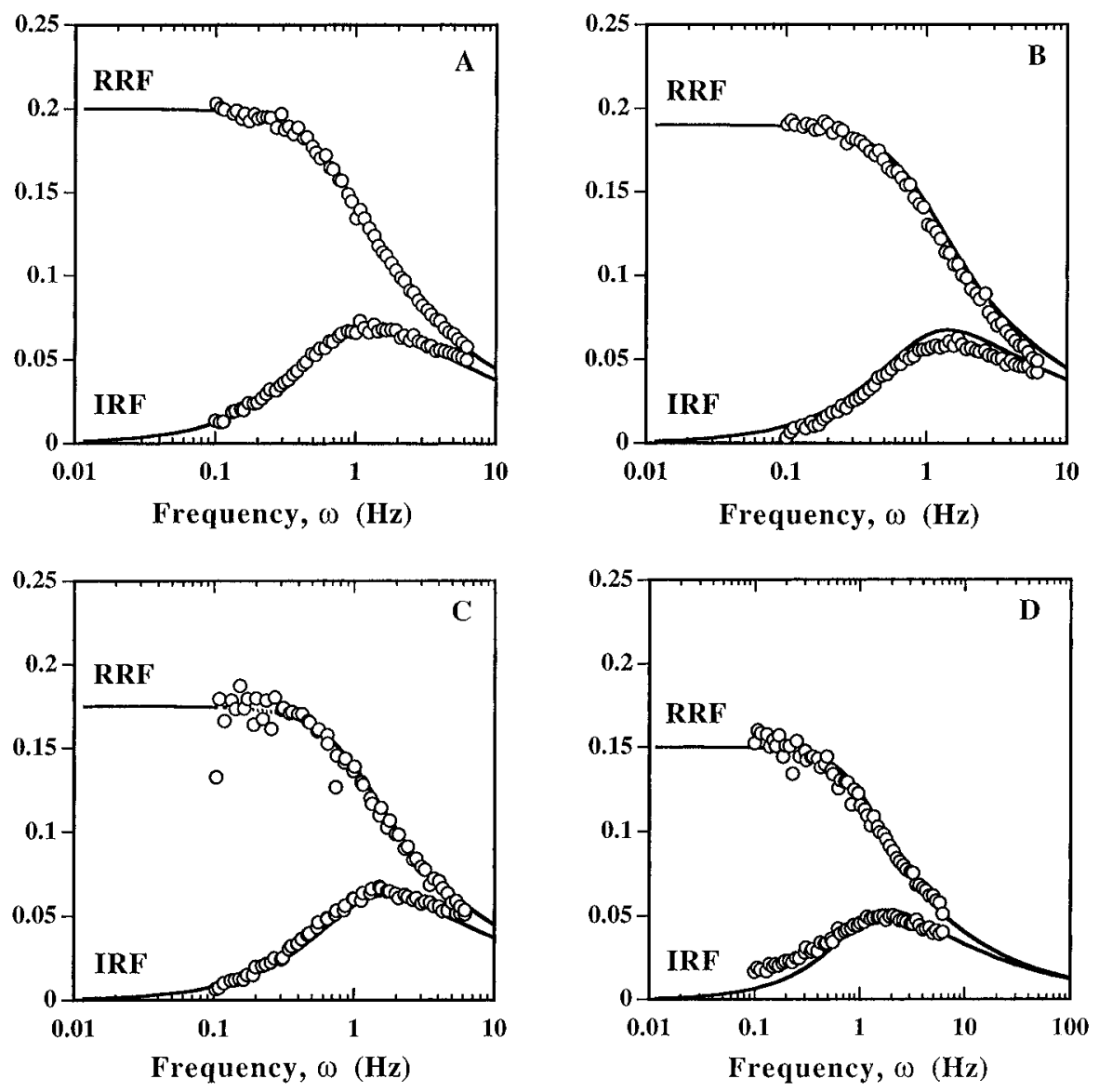

Figure 6. RRF and IRF components for $\mathrm{N}_{2}$ on silica particles at $2.13 \mathrm{kPa}$ and various temperatures: (A) $308 \mathrm{~K}$; (B) $348 \mathrm{~K}$; (C) $398 \mathrm{~K}$; (D) 573 K.

TABLE 1: Adsorption Capacities and Diffusivity Constants for $\mathrm{N}_{2}$, Xe, and Isobutane at $2.13 \mathrm{kPa}$ and Various Temperatures

\begin{tabular}{|c|c|c|c|c|c|c|}
\hline \multirow[b]{2}{*}{$T(\mathrm{~K})$} & \multicolumn{2}{|c|}{ nitrogen } & \multicolumn{2}{|c|}{ xenon } & \multicolumn{2}{|c|}{ isobutane } \\
\hline & $K_{\mathrm{s}}$ & $D / R_{0}^{2}\left(\mathrm{~s}^{-1}\right)$ & $K_{\mathrm{s}}$ & $D / R_{0}^{2}\left(\mathrm{~s}^{-1}\right)$ & $K_{\mathrm{s}}$ & $D / R_{0}^{2}\left(\mathrm{~s}^{-1}\right)$ \\
\hline 308 & 0.058 & 0.140 & (2) & 0.061 & 1.558 & 0.097 \\
\hline 348 & 0.048 & 0 & 0.109 & 0.073 & 0.600 & 0.103 \\
\hline 398 & 0.033 & 0.166 & 0.082 & 0.076 & 0.250 & 0.110 \\
\hline 573 & 0.008 & 0.192 & 0.045 & 0.083 & 0.140 & 0.133 \\
\hline
\end{tabular}

$\left(K_{\mathrm{g}}=V_{\mathrm{p}} / V_{\mathrm{e}}=0.142\right)$ calculated from independent measurements of the pore volume of the silica particles $\left(V_{\mathrm{p}}=19.14\right.$ $\mathrm{cm}^{3}$ and $V_{\mathrm{e}}=134.74 \mathrm{~cm}^{3}$ ). For a given amount of sample, the value of $K_{\mathrm{g}}$ should remain unchanged and equal to 0.142 at all temperatures. Thus, the height of the RRF components, which exceeds $K_{\mathrm{g}}$ below $573 \mathrm{~K}$, and the diffusion-like characteristics of the data suggest that the quasi-equilibrated physisorption process occurs concurrently with diffusion. As a result, the solid lines in Figure 6 were obtained by a nonlinear least-squares fitting of the data using eqs 16 and 17; the only adjustable parameters are the total capacity $\left(K^{\mathrm{app}}\right)$ and the apparent diffusion coefficient $\left(D^{\mathrm{app}}\right)$. The agreement between data and theory is excellent at all temperatures. The greater scatter at $573 \mathrm{~K}$ reflects the less accurate temperature control achieved by the resistive heating of the cell used in our experiments. Columns 2 and 3 in Table 1 summarize the adsorption capacities $\left(K_{\mathrm{s}}\right)$ and diffusion constants $\left(D / R_{0}{ }^{2}\right)$ obtained from the fitting procedure. The values of $K_{\mathrm{s}}$ and $D$ were obtained from their apparent values using eq 17.

The nitrogen adsorption capacities reported in Table 1 are extremely small and correspond to very small fractional coverages on the silica particles. Yet, the adsorbed amounts are comparable to the amount of gas that penetrates the pore volume during one fluctuation (relative capacities of $0.058 \mathrm{vs}$ 0.142 at $308 \mathrm{~K}$, respectively). At $308 \mathrm{~K}$ and $2.13 \mathrm{kPa}$, the pore volume exchange is $0.232 \mu \mathrm{mol}\left[\left(P_{\mathrm{e}} V_{\mathrm{p}} / R T\right)\left(\Delta V / V_{\mathrm{e}}\right)\right]$ and the adsorption exchange is only $0.095 \mu \mathrm{mol}$. In terms of a surface concentration, the latter amount corresponds to a fractional coverage of $2.75 \times 10^{-6}$, assuming $16.2 \AA^{2}$ for the crosssectional area of physisorbed nitrogen. ${ }^{14}$ According to eq 17, this small but rapid adsorption process gives an apparent diffusion coefficient that is $27.5 \%$ smaller than its true value at $308 \mathrm{~K}$. These corrections are relatively small for $\mathrm{N}_{2}$ diffusion in silica but become increasingly important for Xe (section 4.2) and for isobutane (section 4.3) at the same conditions. This discussion illustrates the importance of accounting for physisorption processes present in diffusion measurements and the very high sensitivity of this frequency modulation technique in measurements of very small changes in surface coverages.

The $\mathrm{N}_{2}$ diffusivities reported in Table 1 follow the $T^{1 / 2}$ dependence expected in the Knudsen diffusion regime. At 2.13 $\mathrm{kPa}$, the mean free path $(l)$ of nitrogen varies from $3310 \mathrm{~nm}$ at $308 \mathrm{~K}$ to $6158 \mathrm{~nm}$ at $573 \mathrm{~K}$, both of which are much larger than the mean pore diameter $(7.0 \mathrm{~nm})$ of the silica particles. As a result, molecules move through the pore structure by successive redirecting collisions against the pore surfaces and the diffusion coefficient is independent of pressure, proportional to the square root of temperature, and inversely proportional to the square root of the molecular weight.

4.2. Diffusion of Xenon in Mesoporous Silica. Effective diffusivities for Xe within silica particles were also measured using frequency-modulated methods. Figure 7 shows the system response of $\mathrm{Xe}$ at $2.13 \mathrm{kPa}$ and various temperatures. The lines correspond to the best fit of the data with parameters $K^{\text {app }}$ and 

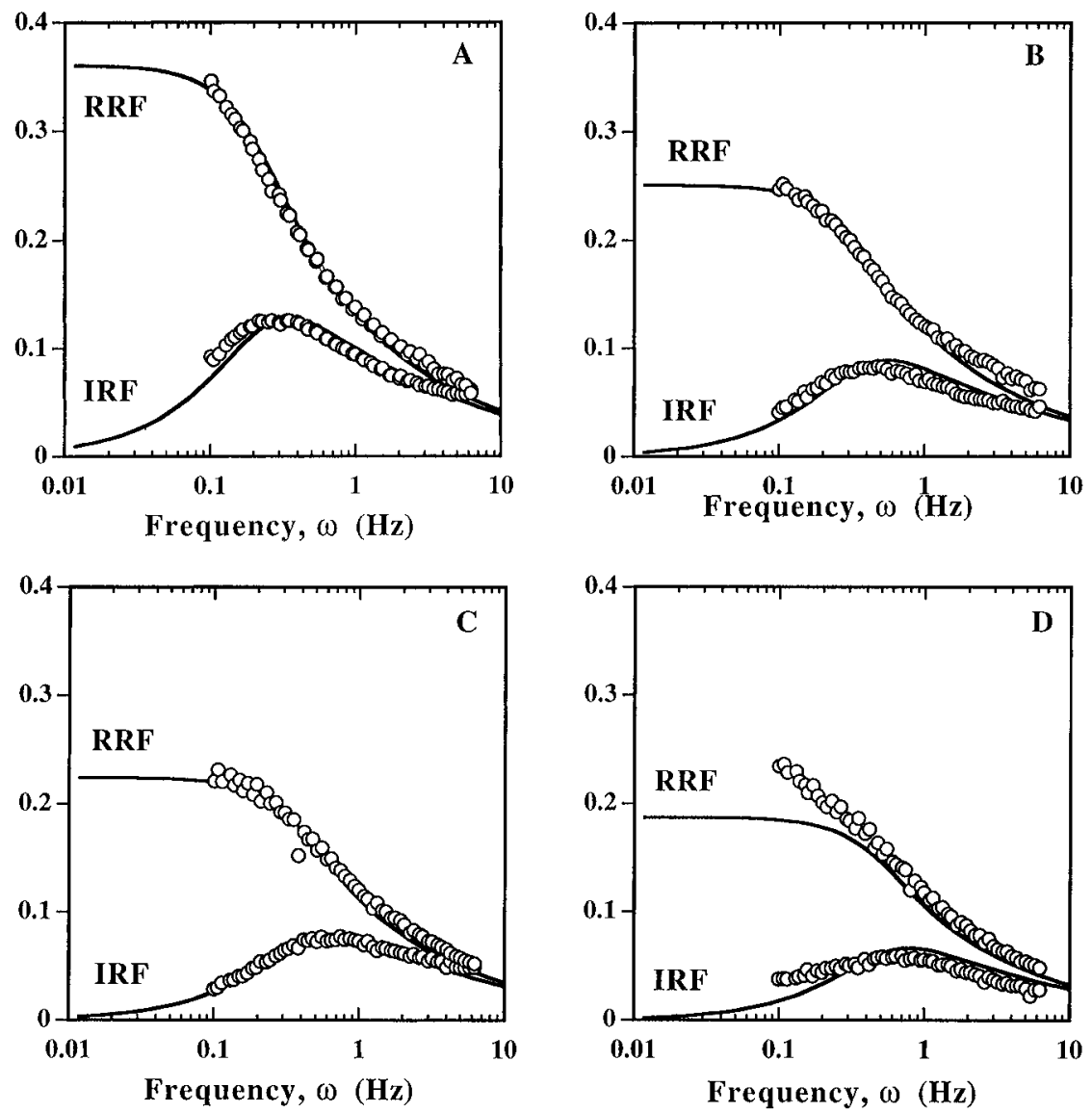

Figure 7. RRF and IRF components for Xe on silica particles at $2.13 \mathrm{kPa}$ and several temperatures: (A) $308 \mathrm{~K}$; (B) $348 \mathrm{~K}$; (C) $398 \mathrm{~K}$; (D) 573 K.

$D^{\text {app }}$ using eqs 16 and 17 . The trends in the results are the same as those for nitrogen. Xe diffusivities are proportional to $T^{1 / 2}$, as predicted by theory (Table 1). A comparison of results between $\mathrm{Xe}$ and $\mathrm{N}_{2}$ shows that experimental diffusivities correctly scale with the inverse of the square root of the molecular weight. At each temperature, Xe adsorbs more strongly than $\mathrm{N}_{2}$. For example, Figure 7D shows that even at $573 \mathrm{~K}$, rapid adsorption of $\mathrm{Xe}$ still occurs during the period of one volume fluctuation; the low-frequency asymptotic limit of the RRF is 0.187 , which exceeds the pore volume capacity of 0.142 (see also Table 1). According to eq 17 and the data in Table 1 , at $308 \mathrm{~K}$, the apparent diffusion coefficient differs by $104 \%$ from it true value.

4.3. Diffusion of $\mathrm{N}_{2}$ /Xe Equimolar Mixtures in Mesoporous Silica. In the Knudsen diffusion regime, molecular collisions occur predominantly with solid surfaces within the pore voids of the material. As a result, individual components in a mixture diffuse independently of each other and are not affected by total pressure. We have carried out frequency modulation experiments to verify that the individual diffusivities for $\mathrm{N}_{2}$ and $\mathrm{Xe}$ in a mixture are consistent with the values obtained previously for the pure components. The experiments were carried out using an equimolar mixture of $\mathrm{N}_{2}$ and $\mathrm{Xe}$ at a total pressure of $2.13 \mathrm{kPa}$ in the same temperature range used for pure $\mathrm{Xe}$ and $\mathrm{N}_{2}$.

Figure 8 shows frequency response data for an equimolar $\mathrm{N}_{2} / \mathrm{Xe}$ mixture at various temperatures. The solid lines were obtained by using the $K^{\text {app }}$ and $D^{\text {app }}$ values of the pure components (Table 1) and assuming that the individual responses are additive. The excellent agreement between the simulations and the experiments shows that $\mathrm{N}_{2}$ and Xe diffuse independently of each other in the Knudsen regime of our experiments.
4.4. Diffusion of Isobutane in Mesoporous Silica. In this section, we examine concurrent isobutane diffusion and adsorption-desorption processes within mesoporous silica particles. Isobutane adsorbs on silica more strongly than $\mathrm{N}_{2}$ and $\mathrm{Xe}$, leading to significant surface coverages at the temperatures of our experiments. Figure 9 shows isobutane adsorption isotherms at various temperatures. The frequency response experiments reported here show that diffusivity corrections due to quasiequilibrated isobutane physisorption become quite large because of the larger adsorption uptakes involved.

Frequency response data for isobutane on silica are shown in Figure 10 at various temperatures. The lines correspond to the least-squares fit of the data using eqs 16 and 17 . The isobutane adsorption capacities $\left(K_{\mathrm{s}}\right)$ and diffusion constants $(D /$ $R_{0}^{2}$ ) are listed in Table 1 . The diffusion coefficients show the correct dependence on temperature and molecular weight, and the adsorption capacities are proportional to the slope of independently measured equilibrium adsorption isotherms at the respective temperatures (see definition of $K_{\mathrm{s}}$ in eq 2) (Figure 9). Figure 10 shows that the low-frequency limits of the RRF components are much larger than those for $\mathrm{N}_{2}$ or Xe molecules (Figures 6 and 7, respectively). For example, at $308 \mathrm{~K}$, the height of the RRF for isobutane is equal to $1.7\left(K_{\mathrm{s}}+K_{\mathrm{g}}=\right.$ $1.558+0.142)$, which translates into a physisorption exchange capacity of about 11 times that of the pore volume. This, in turn, implies that the apparent diffusion coefficient differs by 8.4 times from the true value reported in Table 1 . This very large difference between true and apparent diffusivities highlights the importance of using correct frequency response data analysis in interpreting diffusion measurements when quasiequilibrated physisorption occurs at the conditions of the experiments. 

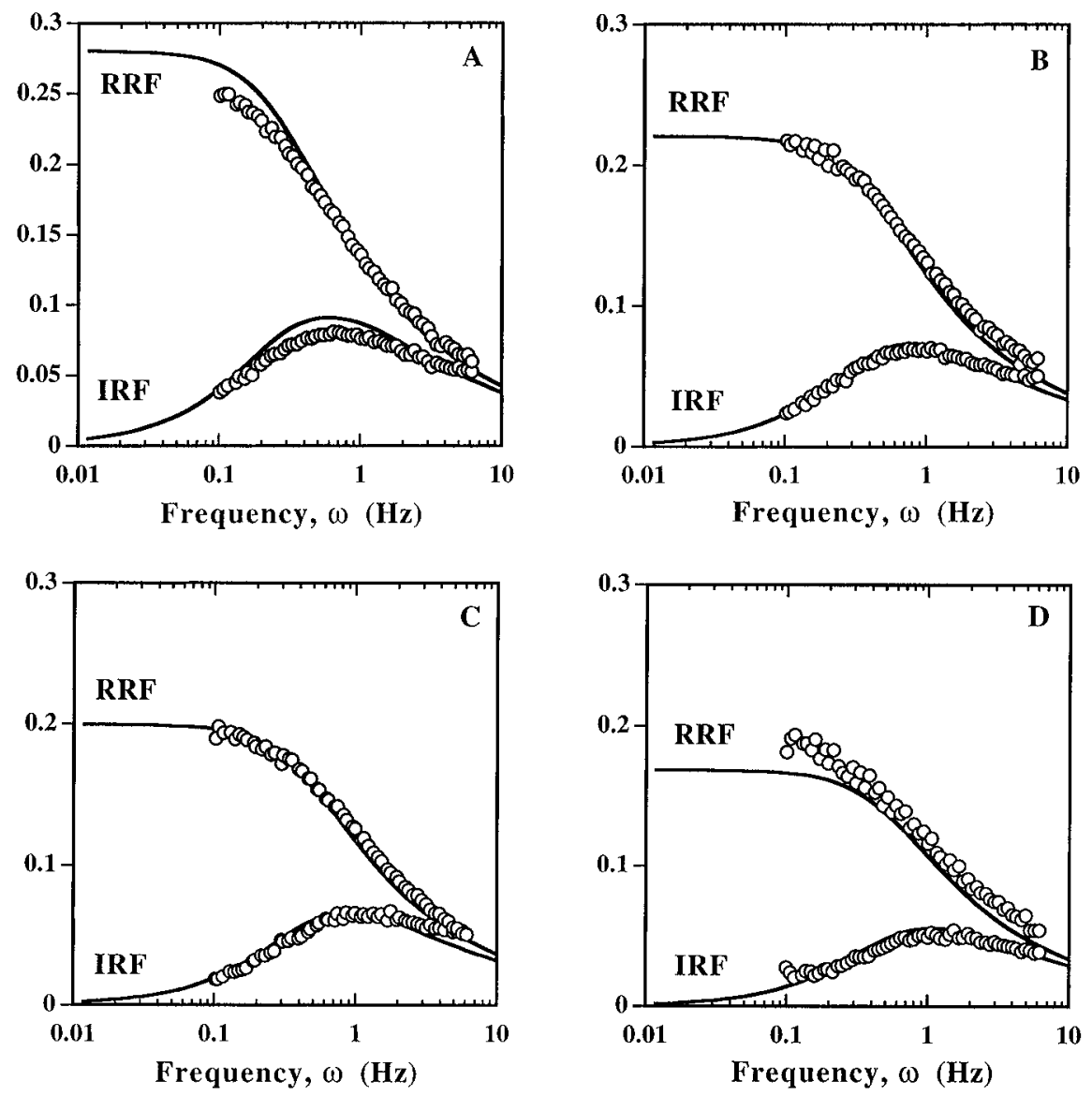

Figure 8. RRF and IRF components for $\mathrm{N}_{2}-$ Xe mixtures on silica particles at $2.13 \mathrm{kPa}$ and various temperatures: (A) $308 \mathrm{~K}$; (B) $348 \mathrm{~K}$; (C) 398 $\mathrm{K}$; (D) $573 \mathrm{~K}$.

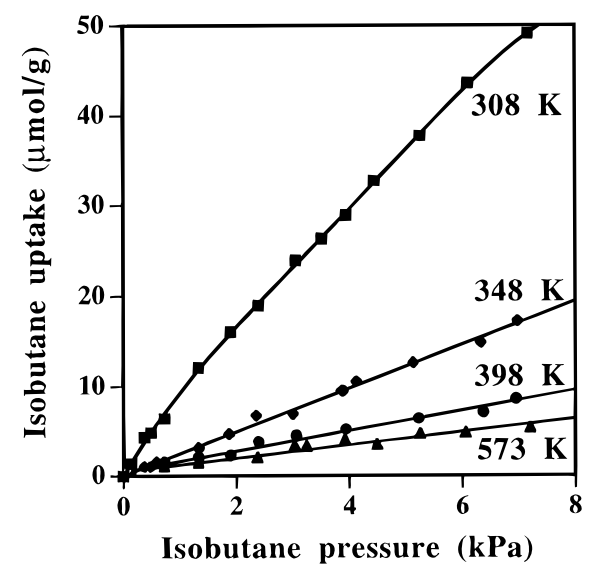

Figure 9. Adsorption isotherms of isobutane on silica at various temperatures.

4.5. Heats and Entropies of Adsorption. Frequency response data for $\mathrm{N}_{2}, \mathrm{Xe}$, and isobutane in silica particles strongly suggest that quasi-equilibrated physisorption occurs concurrently with diffusion even at high temperatures. Here, we extract enthalpies and entropies of adsorption for the various gases from these measurements and compare them to available theoretical and experimental values. Given that both $\mathrm{N}_{2}$ and $\mathrm{Xe}$ adsorb in small quantities and that isobutane isotherms are linear (Figure 9), their adsorption behavior is well described by the low coverage limit of adsorption isotherms. Thus, based on the $K_{\mathrm{s}}$ values from Table 1, we have calculated the adsorption equilibrium constant $H$ (or Henry's law constant) at the various temperatures. As predicted by the van't Hoff equation, ${ }^{13}$ a plot of $\ln (H)$ vs $1 / T$ should yield a straight line with slope $-\Delta H_{\text {ads }} / R$ and intercept $\Delta S_{\text {ads }} / R$, where $\Delta H_{\text {ads }}$ and $\Delta S_{\text {ads }}$ are the enthalpy and entropy of adsorption, respectively. To evaluate the adsorbed concentration at saturation, we have used molecular cross-sectional areas of $16.2,23.2$, and $50.7 \AA^{2}$ for $\mathrm{N}_{2}$, Xe, and isobutane, respectively. ${ }^{14}$ Figure 11 shows the expected linear behavior when $\ln (H)$ is plotted against $1 / T$. As mentioned earlier in the analysis of the RRF and IRF functions, because our experiments are less reliable at $573 \mathrm{~K}$, we have not included this data point in Figure 11. The data in Figure 11 are consistent with enthalpies of adsorption of $-2.2,-3.4$, and $-5.6 \mathrm{kcal} /$ mol for $\mathrm{N}_{2}, \mathrm{Xe}$, and isobutane, respectively. These values are in the range expected for physisorption processes of small molecules on surfaces. ${ }^{15-17}$ Also, Figure 11 leads to entropies of adsorption of $-16.4,-17.1$, and $-18.9 \mathrm{cal} /(\mathrm{mol} \mathrm{K})$ for $\mathrm{N}_{2}$, $\mathrm{Xe}$, and isobutane, respectively. These entropies of adsorption are in excellent agreement with those measured experimentally by other techniques. They are also consistent with the difference in entropy associated with the change from a three-dimensional gas (Sackur-Tetrode equation) to a perfect two-dimensional gas. ${ }^{15}$ Thus, the values of $\Delta H_{\text {ads }}$ and $\Delta S_{\text {ads }}$ obtained in this section further confirm and characterize the physisorption processes detected by our frequency response experiments.

\section{Conclusions}

This study presents a new application of frequency-modulated perturbation methods to the measurement of dynamic parameters and capacities for coupled diffusion and adsorption within mesoporous solids. In particular, it addresses the diffusion of $\mathrm{N}_{2}, \mathrm{Xe}$, and isobutane in silica particles at conditions where small but measurable physisorption uptakes occur concurrently. These measurements required a wide frequency range and the development of theoretical response functions that rigorously account for the dynamic coupling between adsorption and 

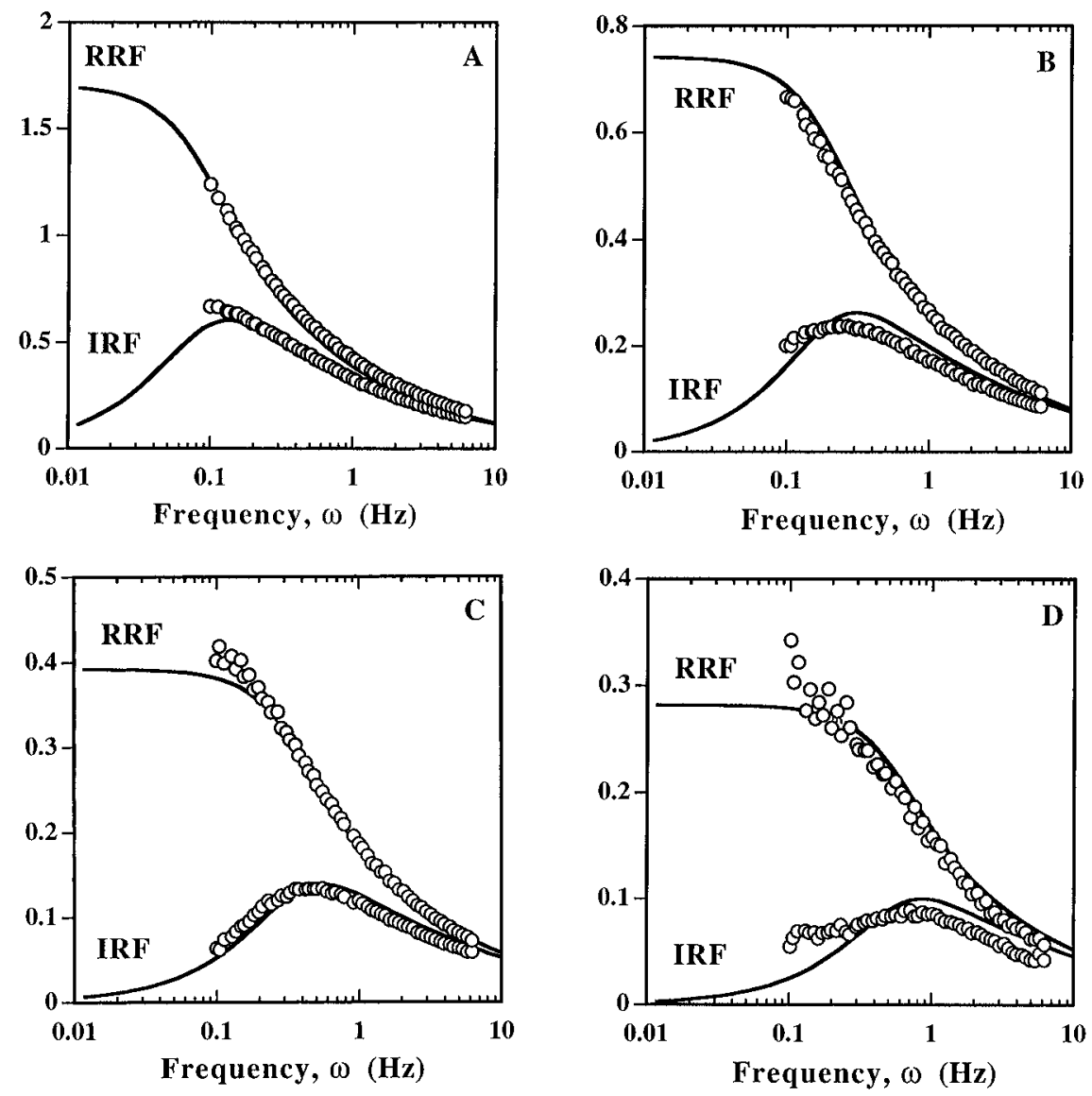

Figure 10. RRF and IRF components for isobutane on silica particles at $2.13 \mathrm{kPa}$ and various temperatures: (A) $308 \mathrm{~K}$; (B) $348 \mathrm{~K}$; (C) $398 \mathrm{~K}$; (D) $573 \mathrm{~K}$.

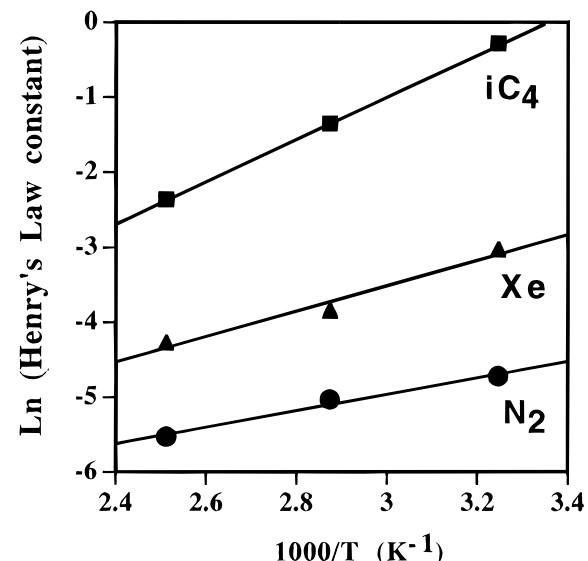

Figure 11. Estimation of heats and entropies of adsorption for $\mathrm{N}_{2}$, $\mathrm{Xe}$, and isobutane using the van't Hoff equation.

diffusion processes. The underlying theory clearly prescribes the corrections that are needed to translate the apparent diffusion coefficients (influenced by adsorption uptakes) into their true values. The measured Knudsen diffusivities correctly scale with temperature and molecular weight, and the adsorption uptakes are well described by Henry's law constants for these gases at the conditions of the experiments. Experimental enthalpies and entropies of adsorption are consistent with theoretical estimates and the results of other studies.

Our continuing research in this area aims to extend the applicability and usefulness of frequency modulation methods by the design of improved perturbation systems that achieve a wider frequency range and that reduce experimental time through the use of multifrequency techniques. We are also developing new transfer functions that rigorously describe the coupling of simultaneous processes that occur at conditions typical of catalysis and membrane separations. Two areas of great interest are the study of surface diffusion processes and the detailed interpretation of frequency response experiments on microporous materials.

\section{References and Notes}

(1) Deisler, P. F., Jr.; Wilhelm, R. H. Ind. Eng. Chem. 1953, 45, 1219.

(2) Naphtali, L. M.; Polinski, L. M. J. Phys. Chem. 1963, 67, 369.

(3) Yasuda, Y. J. Phys. Chem. 1976, 80, 1867.

(4) Marcelin, G.; Lester, J. E.; Mitchell, S. F. J. Catal. 1986, 102, 240.

(5) Bulow, M.; Schlodder, H.; Rees, L. V. C.; Richards, R. E. Stud. Surf. Sci. Catal. 1986, 28, 579.

(6) Li, Y. E.; Willcox, D.; Gonzalez, R. D. AIChE J. 1989, 35, 423.

(7) Schrieffer, J. R.; Sinfelt, J. H. J. Phys. Chem. 1990, 94, 1047.

(8) Yasuda, Y. J. Phys. Chem. 1982, 86, 1913.

(9) Reyes, S. C.; Iglesia, E. In Catalysis; Spivey, J. J., Agarwal, S. K., Eds.; Vol. 11, Specialist Periodical Reports; Royal Society of Chemistry: 1994; pp 51-92.

(10) Reyes, S. C.; Iglesia E. J. Catal. 1991, 129, 457.

(11) Yasuda, Y.; Sugasawa, G. J. Catal. 1984, 88, 530.

(12) Froment, G. F.; Bischoff, K. B, Chemical Reactor Analysis and Design; John Wiley and Sons: New York, 1990.

(13) McClellan, A. L.; Harnsberger, H. F. J. Colloid Interface Sci. 1967, $23,577$.

(14) Ruthven, D. M. Principles of Adsorption and Adsorption Processes; John Wiley and Sons: New York, 1984.

(15) Kemball, C. Adv. Catal. 1950, 2, 233-250.

(16) Cassel, H. M.; Neugebauer, K. J. Phys. Chem. 1936, 40, 523.

(17) Adamson, A. W. Physical Chemistry of Surfaces; John Wiley and Sons: New York, 1990.

(18) Van-den-Begin, N. G.; Rees, L. V. C. Zeolites: Facts, Figures, Future; Jacobs, P. A., van Santen, R. A., Eds.; Elsevier Science Publishers: New York, 1989; pp 915-924.

(19) Jordi, R. G.; Do, D. D. Chem. Eng. Sci. 1993, 48, 1103. 